\title{
¿Unas elecciones de verdad? Autenticidad, representación y conflicto en los concursos de Reinas Indígenas de Guatemala
}

True elections? Authenticity, Representation and Conflict at the Indigenous Queens Pageants in Guatemala

De vraies élections? Authenticité, représentation et conflit dans les concours de reines indigènes au Guatemala

\section{Gemma Celigueta}

\section{OpenEdition}

\section{Journals}

Edición electrónica

URL: https://journals.openedition.org/jsa/14952

DOI: $10.4000 /$ jsa. 14952

ISSN: $1957-7842$

Editor

Société des américanistes

Edición impresa

Fecha de publicación: 15 junio 2017

Paginación: 27-49

ISSN: 0037-9174

Referencia electrónica

Gemma Celigueta, «¿Unas elecciones de verdad? Autenticidad, representación y conflicto en los concursos de Reinas Indígenas de Guatemala», Journal de la Société des américanistes [En línea], 103-1 | 2017, Publicado el 15 junio 2017, consultado el 02 septiembre 2022. URL: http:// journals.openedition.org/jsa/14952 ; DOl: https://doi.org/10.4000/jsa. 14952 


\title{
¿Unas elecciones de verdad? Autenticidad, representación y conflicto en los concursos de Reinas Indígenas de Guatemala
}

\author{
Gemma Celigueta *
}

En este artículo me interesa analizar las diversas maneras de representar la autenticidad indígena a través de los concursos de Reinas Indígenas de Guatemala. El análisis permite mostrar cómo se ha representado lo indígena o lo maya en este país. He escogido reflexionar sobre esta diversidad a través de los conflictos y debates que despiertan estos concursos en relación con su autenticidad. Mediante el análisis queremos plantear la relación existente entre una representación " estética » y una representación «política » de lo indígena. [Palabras clave: Pueblos Indígenas, concursos de belleza, Guatemala, representación, autenticidad.]

True elections? Authenticity, Representation and Conflict at the Indigenous Queens Pageants in Guatemala. In this article I will delve into de history of the Indigenous Queens Pageants in Guatemala. The analysis reflects on different ways of representing what is Indigenous and what is Maya in this country. My focus will be on the conflicts and debates these contests arouse in relation to their authenticity. I want to question the relationship between an « aesthetic » representation and a « political » representation of the Indigenous People. [Key words: Indigenous peoples, beauty pageants, Guatemala, representation, authenticity.]

De vraies élections? Authenticité, représentation et conflit dans les concours de reines indigènes au Guatemala. Dans cet article je m'intéresse à l'histoire des concours de reines indigènes du Guatemala. Mon analyse montre les différentes façons de représenter ce qui est indigène et ce qui est maya dans ce pays. J'ai choisi de réfléchir sur cette diversité à travers les conflits et les débats que suscitent les concours à propos de leur authenticité. L'interrogation porte sur la relation entre une représentation " esthétique » et une représentation "politique » de l'indigène. [Mots-clés: Peuples Indigènes, concours de beauté, Guatemala, représentation, authenticité.]

* Departament d'antropologia Social, Universitat de Barcelona [gceligueta@ub.edu]. 


\section{Introducción}

Hace ya algunas décadas que los concursos para elegir a Reinas Indígenas - o Representativas Mayas o Indígenas como prefieren llamarse ahora - se han convertido en parte esencial de los programas de las fiestas patronales y/o de las fiestas patrias de casi todos los pueblos de Guatemala. Proliferan, además de estos concursos locales, concursos temáticos ${ }^{1}$, por áreas lingüísticas ${ }^{2}$, departamentos ${ }^{3}$ o de ámbito nacional ${ }^{4}$ e incluso internacional, como la elección de la Princesa Ixchel que, desde el año 2011, tiene lugar en Florida (EEUU) entre las hijas de algunos migrantes indígenas guatemaltecos (Molina 2012, p. 121-122). Si a ello añadimos los concursos de niñas indígenas ${ }^{5}$ que se están expandiendo desde los años 2000, o bien los que también se organizan en las aldeas de algunos municipios, podemos considerar que, a pesar de su apariencia frívola y superficial, estos eventos han adquirido un significativo valor social en Guatemala.

Ciertamente su abrumadora presencia no es del gusto de todo el mundo. Como señalan la mayoría de los autores, los concursos han sido y son criticados por algunos actores de Guatemala por no ser expresiones culturales auténticas de los pueblos indígenas (Molina 2012, p. 97, 99; Celigueta 2014, p. 74; Schackt 2005, p. 273, 284), por mostrar una visión tergiversada, turística y comercializada de la cultura maya (Schackt 2005, p. 273) o bien por faltarle el respeto a los pueblos indígenas en general y a la mujer maya en particular al exhibirla como a un objeto (López García 2015, p. 188; Molina 2012, p. 99).

1. Por ejemplo, el concurso de Nim Ali Rech Tinimit Rech Kakaw (Reina Nacional del Cacao), que elige a la representativa de las regiones productoras de cacao desde el 2010 (Molina 2012, p. 106).

2. Por ejemplo, Ka'tu Suckchij (Flor del Maguey) como representativa del área ch'orti' desde 1985, Rukotz'i'j Kaqchikel Tinamit (Flor del pueblo Kaqchikel) desde 1985 o Rixq'uun Poqom (Princesa Poqom) del área poqomchi' (Molina 2012, p. 104-106).

3. Por ejemplo, el concurso de Princesa Tezulutlán en el departamento de Alta Verapaz desde 1969, Princesa Xinabajul en el departamento de Huehuetenango desde los años 1970, Nim Ali Re Xochiltepetl (Gran Muchacha de Suchitepéquez) desde 1977, Ixkik Uk'ux K'iche' (Muchacha Alma de la Cultura K'iche') en el departamento del Quiché o Rum 'ial Tinamital B'oko (Hija del departamento de Chimaltenango) desde el 2012 (Molina 2012, p. 101-104).

4. Además del concurso de Rab 'in Ajaw (Hija del Rey), que desde 1971 elige en Cobán a una representativa indígena a nivel nacional, se celebran los concursos nacionales de Rumi'al Yum Kax (Hija del Maíz) en Chimaltenango desde 1989, Reina Diosa Ixchel en Suchitepéquez desde 1993, Tixel Tenamit (Tenanza o mujer de la cofradía del pueblo) en San Pedro Sacatepéquez desde 1998, Reina Indigena Nacional Cotz'i’j Iximulew en Chichicastenango desde el 2000, Uko tzijal Ri Maya Tinamit Re Paxil Kayala (Flor Nacional del Pueblo Maya) en Quetzaltenango desde el 2005 o Princesa de los Cuatro Puntos Cardinales en San Lucas Sacatepéquez desde el año 2007 y en la Antigua Guatemala desde el 2011 (Molina 2012, p. 106-121).

5. Como el concurso de Alaj Ukotzij Tinimit Re Xelajuj Noj (Pequeña Flor del Pueblo de Quetzaltenango), que tiene lugar desde el 2005 o el de Ri' Man Ajaw (Nieta del Rey) en Cobán (Molina 2012, p. 122). 
En la calle, en algunos artículos de la prensa o en las mismas redes sociales podemos apreciar críticas similares. Una joven k'iche', por ejemplo, escribía en su perfil de Facebook, en 2015: « Sigo creyendo que todo esto de las Reinas es para distraer al pueblo de hacer algo verdadero. » Yo misma tuve serias dudas antes de prestarle atención académica a estos concursos y sólo lo hice cuando la historia del Xel-ju, la organización política indígena que estaba estudiando en la ciudad de Quetzaltenango, en el altiplano occidental de Guatemala, me llevó directamente hasta el concurso de Reina Indígena de Xelajú ${ }^{6}$. En el pasado, los clubes que postulaban candidatas a Reina se habían comportado como partidos políticos, haciendo campaña electoral por aspirantes que hasta 1969 eran elegidas mediante el voto popular en las urnas. Dichos clubes han sido considerados no sólo como precedentes del Xel-ju, sino también de las futuras organizaciones del movimiento indígena (Celigueta 2012, 2014).

En realidad, estos eventos son tomados bastante en serio por parte de la población. Prueba de ello es la emoción que despierta entre el público la aparición de las jóvenes en los escenarios, el apoyo de los grupos postulantes a su candidata con pancartas, silbatos, serpentinas y cánticos, el seguimiento de los medios de información, el lleno total de los espacios en donde se eligen a las futuras Reinas o bien las fotografías y recuerdos del reinado que inundan las casas de las antiguas Reinas. En la misma Quetzaltenango, una ciudad en la cual la memoria de su importante historia indígena - por no hablar de las mujeres - es poco visible, existe un parque de juegos infantiles con el nombre de la Reina de 1970, así como también un busto de la primera Reina Indígena Rosa de Paz Chajchalaj (1934-1935) junto a una placa conmemorativa con el nombre de todas las Reinas de la ciudad. Por si fuera poco, la comisión organizadora del concurso en 1984 y la Umial Tinimit Re Xelajuj Noj (Hija del pueblo de Quetzaltenango) de 2003-2004 han editado respectivamente dos publicaciones con los nombres, fotografías, biografía y actividades realizadas durante cada reinado (Tzunum 1985; López Quemé 2004). Podemos valorar negativamente el hecho de que estos concursos hayan sido uno de los pocos espacios de visibilidad de las mujeres indígenas, pero ciertamente no podemos ignorarlos.

Es por estas razones que estos eventos han llamado la atención de varios autores nacionales y extranjeros que han clasificado y catalogado la diversidad de concursos existentes en el país (Molina 2012), han recogido la historia de algunos de ellos como el de Quetzaltenango (Tzunum 1985; López Quemé 2004; Celigueta 2012, 2014) o han analizado el importante papel político de las Reinas Indígenas del siglo XXI (López García 2015). Pero probablemente, el

6. Podemos encontrar escrito el nombre k'iche' de Quetzaltenango como Xelajú, Xelajuj, Xelajuj Noj o Xelajuj No'j, dependiendo de si se aplica o no la normalización lingüística. En este artículo he escogido escribirlo en cada ocasión según aparece en las fuentes, porque refleja el momento que atraviesa el concurso. 


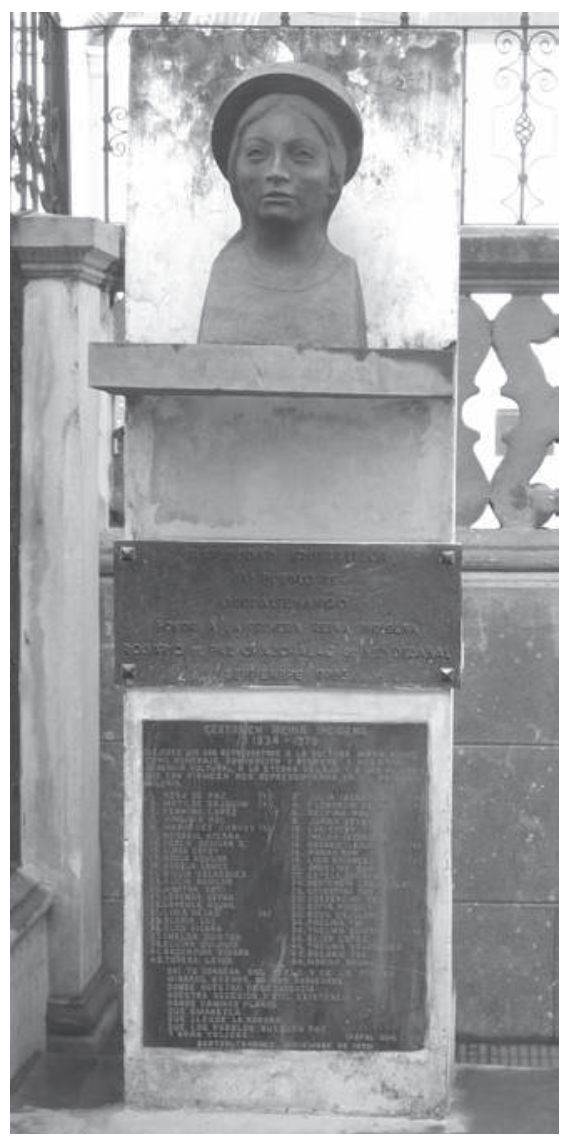

Fig. 1 - Homenaje a las Reinas Indígenas situado en la entrada del teatro municipal de Quetzaltenango. Fotografía de la autora, Agosto del 2006.

tema que más se ha problematizado en relación con las Reinas Indígenas de Guatemala ha sido el de la « autenticidad » (McAllister 1996; Konefal 2009; Schackt 2005). Como afirma Handler (1986), la autenticidad es una construcción cultural ligada a una noción de cultura como entidad única e individualizable. La existencia de una colectividad nacional depende, en cierta manera, de la posesión de esa cultura única y original. Por ello, al igual que en otros países de América Latina, las autoridades guatemaltecas buscaron distinguirse del resto de naciones a partir de su herencia indígena. Por ello, también, el movimiento indígena primero y maya después cuestionaron estas representaciones nacionales proponiendo modelos alternativos de lo indígena que han ido cambiando según contextos e intereses. La « indianidad», « indigeneidad » $\mathrm{o}$ « mayanidad » son performativas (Canessa 2012). Son encarnadas y representadas por individuos que dialogan de esta manera con representaciones defendidas por el Estado o por el movimiento indígena. El desafío es que, al mantener este diálogo en términos de autenticidad - es decir en términos unívocos y esenciales -, dichas representaciones suelen entrar en conflicto.

En este artículo quiero aportar ejemplos históricos y etnográficos de esta variedad de significados de lo indígena. He escogido mostrar esta diversidad a través de los conflictos y debates que provocan los concursos de Guatemala y que a menudo están relacionados con la autenticidad de los mismos. La mayoría de observaciones y entrevistas se realizaron en 1998, 1999 y 2006, durante mi trabajo de campo en Quetzaltenango para la tesis doctoral, aunque he realizado estadías más cortas en el 2009, 2010 y 2016 relacionadas con otras investigaciones. Como la elección de Umial Tinimit se realiza a finales de agosto, precediendo a las actividades de la Feria de la Independencia y coincidiendo también con mis vacaciones académicas, en mis viajes a Guatemala suelo coincidir con actividades asociadas con estos eventos. He asistido a la velada de elección de la Umial Tinimit de 1999 en el teatro municipal, a diversas presentaciones de las candidatas en el parque central de 
la ciudad, a varios talleres impartidos a las concursantes por las organizaciones mayas y he seguido también la elección de $U$ mial Tinimit en la televisión local. Además, he asistido a los concursos de otros pueblos como por ejemplo Cantel, en el mismo departamento de Quetzaltenango. Quetzaltenango o Xelajuj Noj en k'iche' está situada en la región más densamente poblada y con más población indígena del país, el altiplano occidental guatemalteco, y destaca por ser el centro administrativo y comercial del mismo. Según el último censo de población (2002), cuenta con 127.000 habitantes, de los cuales 63.714 se clasifican como indígenas y 63.855 como no indígenas. La mayoría de estos indígenas pertenecen al grupo maya-k'iche', viven en el centro urbano y han sido considerados como una clase media o una pequeña burguesía indígena (Velásquez 2002).

Los concursos de Quetzaltenango proporcionan pues mi principal material de reflexión, aunque en este artículo busco generalizar el análisis dialogando con otros autores (McAlllister 1996; Molina 2012; Schackt 2005; López García 2015; Konefal 2009) que también han prestado atención a este tipo de eventos en Guatemala, especialmente el de Rab ’in Ajaw en Cobán. Además de contar con abundante material sobre el mismo, el concurso de Quetzaltenango cuenta con la ventaja de ser uno de los más antiguos y conocidos del país. Tanto es así que el 18 de octubre del 2011 se declaró como patrimonio cultural intangible de la Nación. Sólo el Festival Folklórico Nacional de Cobán, cuya actividad principal es la elección de Rab'in Ajaw, ha recibido esa distinción. Al reflexionar a partir de este concurso, cuento asimismo con la ventaja de cierta perspectiva histórica que me permite observar las transformaciones de este tipo de eventos, aunque es importante aclarar que cada localidad tiene su propio ritmo y contexto.

\section{De Indias Bonitas a Reinas y Princesas Indígenas. Belleza, Etnicidad y Nación}

Los primeros concursos de belleza indígena de los que se tiene noticia en Guatemala datan de la década de 1930. Se trata de los concursos de India Bonita Cobanera en 1931 (McAllister 1996, p. 112), de India Bonita de Quetzaltenango hacia finales de los años 1920 (Tzunum 1985, p. 3) y de India Bonita del departamento de Sacatepéquez en 1933 (Molina 2012, p. 92). Al parecer, los concursos de belleza indígena llegaron a Guatemala desde el México revolucionario. En 1921, el gobierno mexicano y dos de los periódicos más leídos del momento organizaron en la capital un concurso de india bonita para elegir a la indígena más bella del país (Ruiz 2001; Molina 2012, p. 92; Konefal 2009, p. 58). Es probable que el concurso se haya difundido rápidamente a otros lugares, alentado por unas autoridades que vieron en él un espacio adecuado para promover el nacionalismo y la hasta entonces fallida integración del indio en la Nación.

En Guatemala, la década de 1930 coincide con la llegada al poder de Jorge Ubico (1931-1944), el último dictador liberal del país antes de la revolución 
democrática de 1944, conocido por promover una política populista, clientelar y paternalista con los grupos indígenas del país (Grandin 2007; Taracena et al. 2002). Entre otras medidas, Ubico promocionó espectáculos y exposiciones folklóricas como por ejemplo los llamados « pueblos indígenas », en los cuales se mostraban a personas de carne y hueso realizando actividades « típicas » como tejer, hacer canastos o tocar la marimba bajo rótulos con los nombres de sus comunidades de origen. Es también en ese entonces que se incrementó el interés por la civilización maya, erigiendo el pasado prehispánico y algunas de sus figuras, como el héroe resistente a la conquista española Tecún Umán, en símbolos específicos de la nación guatemalteca (Taracena 2002 et al., p. 107-126). Así, mientras por un lado se construía una idea de nación mediante imágenes del pasado prehispánico, por el otro se hacía necesario replantear el lugar y la relación con los indígenas del presente (López García, Celigueta y Mariano 2015, p. 162-165). En este sentido, los concursos de india bonita, en tanto que actos folklóricos, tendieron un puente entre pasado y presente indígena. Escenificaron una solución, ampliamente difundida por la prensa, que intentaba resolver el difícil equilibrio entre el deseo de ser una nación atractiva y moderna y la necesidad de mantener una tradición propia en base al pasado indígena (Ruiz 2001).

Según Cohen et al. (1995, p. 3-5), aunque los concursos de belleza pueden remontarse a la Antigüedad y especialmente a la Edad Media, en la cual se elegían a las reinas de mayo en el marco de las ferias medievales, su expansión está ligada al surgimiento de las naciones modernas. Según estos autores, en los años 1920 y 1930 se difundieron especialmente desde los EEUU hacia los países del sur impulsados por la descolonización y el nacionalismo asociado con este proceso. Hollywood y la prensa ayudaron en su difusión, homogeneizando su estructura. Los concursos de india bonita parecen responder por lo tanto a una doble influencia que es necesario diferenciar. Por un lado, las reinas de las fiestas patronales y las ferias, fuertemente asociadas con una localidad concreta y por el otro, las mises regionales, nacionales e internacionales, cuyo objetivo consiste en promover la unidad de la nación frente a la diversidad local existente (Kite 2014, p. 20). A esta doble influencia cabe añadir una tercera, propiamente indígena, que se iría desarrollando a lo largo del siglo Xx. Aunque estos concursos fueron introducidos y promovidos por autoridades e instituciones ladinas ${ }^{7}$, pronto se convirtieron en una plataforma privilegiada de debate - con símbolos y discursos - sobre la cuestión indígena en la Nación. Por ello, autoridades, líderes y organizaciones indígenas lucharían por su representación en estos escenarios. Un ejemplo temprano lo encontramos en Quetzaltenango.

7. El término clasificatorio « ladino », utilizado en Guatemala para designar a la población que no es indígena, sugiere más bien una identidad cultural hispanizada que un mestizaje biológico aunque su significado cambia con el tiempo y el contexto en el que se usa. Actualmente se observa cierta tendencia a usar el término como si fuera un pueblo o grupo étnico más de Guatemala. 
Durante la Feria Centroamericana de la Independencia, celebrada en la ciudad desde 1884, se acostumbraba a elegir a una belleza local entre las hijas de las familias de la élite ladina. En algún momento, suponemos que en la década de 1920, los organizadores comenzaron a escoger a una « india bonita » entre las hijas de las familias k’iche' más prestigiosas de la ciudad (Tzunum 1985, p. 3). Sin embargo, la India Bonita no era elegida por indígenas ${ }^{8}$, no ostentaba su cargo más allá del período de la Feria y, sobre todo, no respondía a la representación que ciertos k'iche'de Quetzaltenango tenían de lo indígena. En 1934, la Sociedad El Adelanto9 ${ }^{9}$ organizó un concurso para elegir a una « representante de su raza » (Tzunum 1985, p. 3). Decidieron que el concurso se llamaría Reina Indígena de Xelajú porque el de India Bonita les parecía poco respetuoso y, lo que es más importante, decidieron que el concurso lo organizarían las autoridades indígenas de Quetzaltenango. También establecieron que las candidatas debían ser originarias y residentes en Quetzaltenango, hijas de padres indígenas y contar entre 18 y 25 años de edad. Además instauraron la elección mediante voto popular en las urnas electorales que montaron en uno de los barrios indígenas de la ciudad (Tzunum 1985; Celigueta 2014).

De esta manera, aunque el concurso respondía a los intereses de un gobierno que promovía la participación de los indios en las fiestas patrias mediante un acto folklórico, la elección y coronación de una Reina permitió, primero, que la élite k'iche' de Quetzaltenango promoviera una representación de lo indígena más acorde a su gusto e interés y segundo, que continuara presentándose - en lugar de los especialistas e ideólogos de la Nación - como la encargada legítima de los asuntos indígenas (Celigueta 2014, p. 65-68). En las antiguas fotografías que nos han llegado de las Reinas de Quetzaltenango (Tzunum 1985; López Quemé 2004) se puede apreciar cómo los miembros de estas élites imaginaron esta representación.

Las Reinas se muestran solemnes, soberanas y orgullosas, vestidas con sus mejores huipiles, adornadas con numerosas joyas y acompañadas de un cetro, una corona y un manto real fabricado con tejidos indígenas. A veces, también se fotografían en el desfile de carrozas de la Feria, sentadas en tronos majestuosos de reminiscencias mayas y rodeadas por una corte de honor; de hecho, las Reinas se parecen más a una bella imagen de la Virgen María que a una guapa Miss Mundo. Podríamos considerar incluso que muestran una belleza « espiritual », a diferencia de los cuerpos medidos y juzgados de los concursos

8. Según uno de los socios de El Adelanto: « En tiempos de Ubico, el alcalde ladino veía entre las indígenas una patoja bonita y consultaba quien era la familia, entonces enviaba una nota oficial al padre de la señorita ».

9. La sociedad de El Adelanto es la primera sociedad de ayuda mutua y beneficencia indígena de Quetzaltenango creada en 1894 por 52 k'iche' de la ciudad, principales y miembros destacados de la sociedad indígena quetzalteca con la finalidad expresa de llevar el progreso a los indígenas, especialmente mediante la educación (Grandin 2007; Quijivix 1998). 


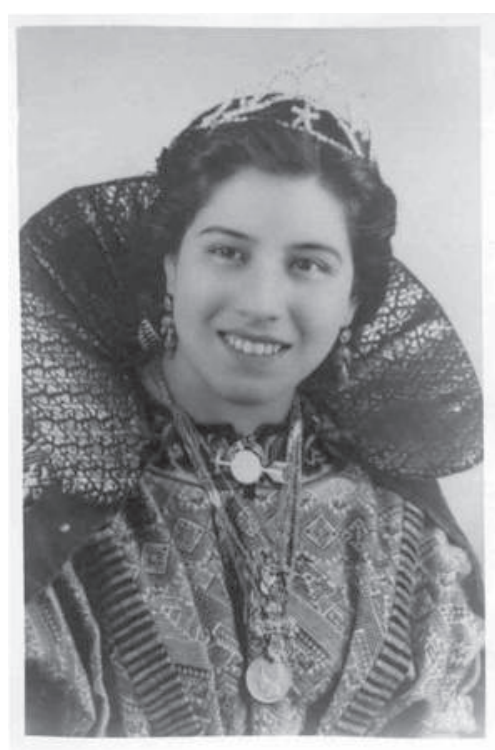

Fig. 2 - Martha Elisa Cotí

López, Reina Indígena de Xelajú 1959-1960. Fotografía cedida por Martha Elisa Cotí.

de belleza a los que estamos acostumbrados actualmente. Esta asociación entre Vírgenes y Reinas es recalcada por un socio de El Adelanto que me explicaba, en 1998, los inicios del concurso: «Las Reinas tenían una corte de honor. Los caballeros de la corte tenían la responsabilidad de llevar en andas a la Reina Indígena como si fuera una Virgen hasta la exposición del Pueblo Indígena ». López García $(2015$, p. 178, 207) también ha destacado este « aire de familia » entre Reinas y Vírgenes, fijándose especialmente en el gusto de ambas por los vestidos ceremoniales y las coronas. Además de estas diferencias con los concursos ladinos, me parece importante subrayar que en ese entonces las Reinas de Quetzaltenango no representaban estampas « típicas » como tejer o hacer tortillas, tal y como se hacía en el caso de los pueblos indígenas de Ubico. Tampoco eran indias bonitas elegidas por un jurado ladino paternalista, racista y condescendiente, tal como refleja la prensa de la época ${ }^{10}$. Incluso en 1951, en medio de las múltiples reformas de la llamada década revolucionaria de Guatemala $^{11}$, la sociedad k'iche' de Quetzaltenango se opuso tajantemente a la propuesta de cambio de nombre del concurso, que durante un año se llamó Flor de Xelajú, para evitar, según las autoridades locales, la discriminación racial implícita en el término « indígena ». Además de reivindicar una identidad diferenciada frente a los discursos igualitarios de la época, es probable también que a la élite k'iche' no le agradara convertir a sus solemnes Reinas en sencillas Flores. Las Reinas de Quetzaltenango fueron probablemente las primeras que intentaron escenificar otra manera " autorizada » de ser indígena dentro del proyecto nacional guatemalteco. Pronto las seguirían otras localidades que convertirían progresivamente a las Indias Bonitas de Guatemala en Princesas y Reinas.

10. «Tarea dura del jurado calificador el de escoger entre tanta belleza indígena. Hay que advertir que en Cobán hay inditas muy bellas producto ya sea de la mezcla de lo sajón y del español, pero esta vez fueron descartadas. La competencia fue de inditas puras » (Nuestro Diario, 6 de agosto de 1936 citado en Molina 2012, p. 93).

11. En octubre de 1944, un movimiento cívico-militar derrocó al gobierno inaugurando un período reformista que duraría hasta 1954. La más conocida de todas fue la reforma agraria de 1952, cuyo ataque a los intereses de la United Fruit Company precipitó la invasión militar desde Honduras con ayuda de la CIA. 


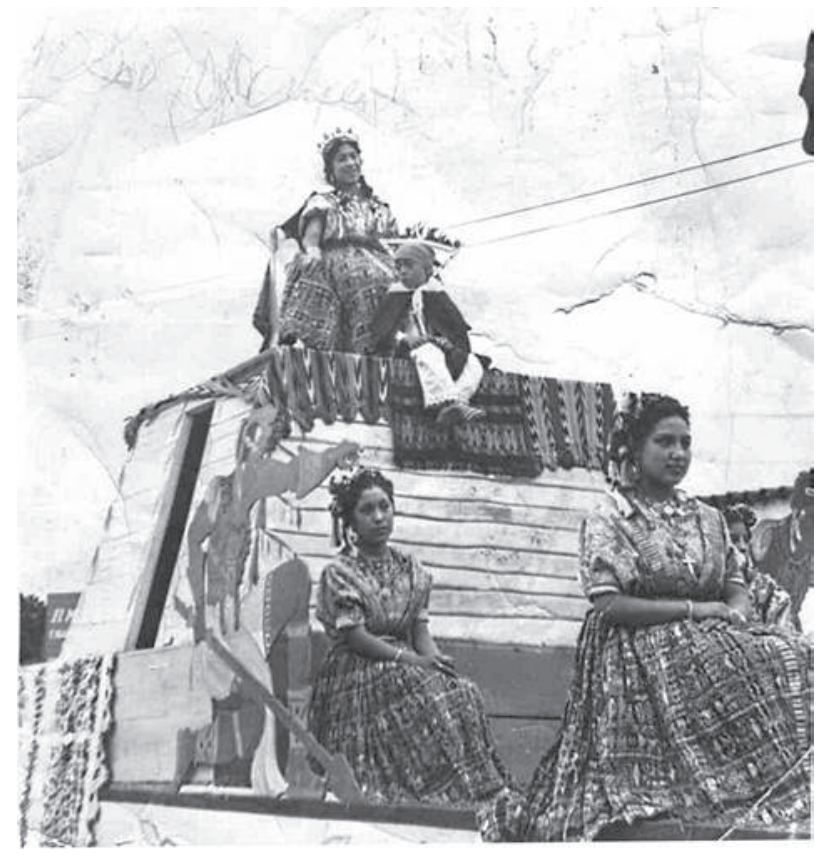

Fig. 3 - Carroza de Maria Concepción Toc Rojas. Reina Indígena de 1962 a 1963.

Fotografía cedida por Martha Elisa Cotí.

\section{De Reinas y Princesas a Hijas del Pueblo. Cuestionando la autenticidad de la representación}

A lo largo de las décadas siguientes, los concursos indígenas se propagaron y consolidaron por todo el territorio, alentados por autoridades, instituciones y organizaciones tanto locales como nacionales, especialmente el Instituto Guatemalteco de Turismo y el Instituto Indigenista Nacional. Además de la prensa escrita, otros medios de comunicación como la radio se sumaron a la transmisión de estos eventos, llegando incluso hasta la población analfabeta. En este sentido, los discursos de las candidatas se fueron convirtiendo en un elemento central de los concursos (Konefal 2009, p. 50). Esta centralidad del discurso puede apreciarse también en las frecuentes fotografías de las décadas de 1960 y 1970, que retratan a las Reinas Indígenas delante de un micrófono (Tzunum 1985). De hecho, las voces de las Reinas acompañaron críticas y demandas de un movimiento indígena que volvía a replantear en otros términos la todavía no resuelta relación de los indígenas con la Nación.

A diferencia de las Indias Bonitas, las Reinas se implicaron progresivamente en actividades realizadas más allá de las ferias y fiestas patronales en las que eran 
elegidas. Durante el año de su reinado organizaban campañas de beneficencia, montaban veladas artísticas e inauguraban obras de infraestructura y monumentos. Además las Reinas y sus acompañantes viajaban por todo el territorio nacional y a veces incluso a otros países, convirtiéndose en « embajadoras » indígenas de sus localidades. Es decir que durante el año de su reinado se las invitaba a asistir a otros eventos culturales - sobre todo a otros concursos de Reina Indígena - como invitadas distinguidas o bien como jurados. A partir de 1971, también viajarían a Cobán para participar en el Concurso de Reina de la Belleza Indígena Nacional, que al año siguiente cambiaría su nombre por el de Rabin Ajau (Hija del Rey), como se lo conoce actualmente (Molina 2012, p. 107). Estos encuentros entre Reinas y acompañantes a lo largo de todo el país les permitieron compartir ideas, experiencias e inquietudes, favoreciendo una identificación más allá de sus comunidades locales que sería muy importante a la hora de articular el movimiento indígena a nivel nacional. Schackt (2005, p. 270) afirma incluso que, en Guatemala, fueron las candidatas de estos concursos las primeras en identificarse como mayas.

Así, en los años 1970, los concursos de Reina Indígena de Guatemala ya se habían convertido en un lugar de encuentro y consolidación de relaciones sociales, en una fuente de legitimidad para la ganadora y su grupo postulante que sería utilizada por el movimiento indígena, en una manifestación autorizada de algunos aspectos de las culturas autóctonas como danzas, trajes e idiomas y en un lugar de aprendizaje para las mujeres que se presentan. Con la articulación y el desarrollo del movimiento indígena, los concursos también se convierten en blanco de críticas y debates sobre la autenticidad de la representación indígena.

Por un lado, se criticaron las representaciones folklóricas promovidas por el Estado y grupos afines en las cuales se valora a un indio de « estampa » sin tener en cuenta la opresión y la exclusión del indio del presente. En este sentido, Konefal (2009) narra la protesta, en 1978, de veintidós Reinas Indígenas contra el concurso de Cobán. En concreto, estas Reinas criticaron en la prensa la contradicción que suponía que estos concursos propusieran homenajear al indígena mientras los indios « genuinos » de Guatemala eran masacrados por el ejército, como había ocurrido recientemente en Panzós ${ }^{12}$. Lo interesante es que, con esta crítica, las Reinas confrontaban la representación que en Cobán se quería dar de lo indígena, " subvirtiendo »-según palabras de Konefal (2009, p. 44) - la noción de autenticidad. Si para los gobiernos militares de Guatemala los campesinos indígenas que protestaban por sus tierras no eran

12. Apenas dos meses antes, el 29 de mayo de 1978, cientos de campesinos ocuparon la plaza mayor de Panzós, un pueblo del mismo departamento que Cobán, protestando por la ocupación de sus tierras. El ejército abrió fuego contra la población matando por lo menos a 35 personas e hiriendo a muchas más. Esta masacre marca el inicio del período más sangriento de la política de « terror » contrainsurgente del estado guatemalteco (Konefal 2009). 
auténticos porque se habían politizado (supuestamente manipulados por grupos de izquierda), algunas Reinas Indígenas que sí habían sido autorizadas como tales ahora homenajeaban y legitimaban a estos indios, a su parecer ejemplares ${ }^{13}$.

Otro ejemplo de cuestionamiento de la autenticidad de la representación lo proporciona la revista Ixim, considerada como una de las revista pioneras del movimiento indígena. En agosto de 1978, el dirigente del Xel-ju Jorge Luís García de León publicó un artículo en esta revista titulado: « El despojo de nuestra identidad en el extranjero por falsos grupos indígenas folkloristas », en el que acusaba a un grupo de danza de Quetzaltenango de representar el folklore indígena sin que ninguno de ellos lo fuera:

Nos indigna que falsos grupos se autodenominen indígenas y se den a la tarea de hablar en nuestro nombre, y lo que es más, se dicen representantes de nuestro folclor. Ya hace algunos años que algunos indios de pura cepa, venimos observando a estos depredadores de nuestra identidad... lo que es peor es que, según sabemos, la Municipalidad de Quetzaltenango, a través de la comisión de reina indígena de Xelajú, ha programado en la segunda parte de la velada, la actuación del ballet folklórico..., esto es lo más absurdo que pueda pensarse, el que en una velada de coronación de una reina indígena, actúe un grupo de farsantes de nuestra cultura... (García de León 1978, p. 6)

La desautorización de los ladinos y sus representaciones de lo indígena se convertirán, hasta el día de hoy, en una de las críticas más recurrentes de los activistas indígenas. Y los concursos serán, de hecho, uno de sus blancos preferidos. Primero, los concursos de señoritas no indígenas que demuestran su « guatemalidad», ya sea con sensuales trajes de fantasía de reminiscencias mayas ${ }^{14}$ o bien con trajes indígenas contemporáneos. Por ejemplo, en el evento de Miss Universo del año 2011, Miss Guatemala usó el traje ceremonial masculino de las cofradías de Chichicastenango, diseñado especialmente para la ocasión; es decir, una sensual y ajustada versión que diferentes organizaciones mayas criticaron por la falta de respeto que suponía para los pueblos indígenas.

13. Me parece importante señalar el papel destacado que parecen haber desempeñado los militares en estos concursos de Guatemala. Otros autores ya han señalado su relación con el de Rab 'in Ajaw en Cobán (Konefal 2009). Tengo conocimiento de que, en Quetzaltenango, la zona militar 17-15 presentó candidatas al concurso por lo menos en 1990, 1996, 1999 y 2000 (López Quemé 2004), aunque seguramente lo hiciera en más ocasiones. En 1999, la candidata de la «zona» tenía una beca de estudios del ministerio de defensa y en su discurso habló de la importancia de la reconciliación. Lo más interesante es que todos mis informantes coincidieron en que era imposible que ganara. Afirmaron rotundamente que jamás había ganado ni ganaría una candidata de la « zona ».

14. Estos trajes de fantasía con numerosas plumas, faldas cortas y bikinis parecen responder a la misma fantasía erótica que Canessa (2008) señala para las siempre blancas Misses de Bolivia: se trata de unir el apreciado cuerpo blanco, considerado por criollos, mestizos y ladinos como más bonito, con la accesibilidad sexual que éstos proyectan a las indias. 
Y en segundo lugar, los propios concursos indígenas que ponen en escena diferentes versiones de lo que es o no auténticamente indígena. Schackt (2005, p. 282-283) proporciona otro ejemplo contemporáneo de conflicto por la representación, cuyo contenido se parece muchísimo al de la revista Ixim: la « Rebelión de las Rabinas » en el 2001, durante el concurso de Rabin Ajau en Cobán. El descontento con la organización del concurso por las malas condiciones de la comida y el alojamiento de las candidatas se materializó en el discurso de la Rabin Ajau saliente, Mercedes Adelina García Marroquín, calentando los ánimos del público. Poco después, un error desafortunado del maestro de ceremonias al anunciar como ganadora a otra muchacha que la elegida por el jurado provocó que ninguna de ellas aceptara el cargo. El descontento del público causó la anulación del concurso y Mercedes acabó depositando la corona en la estación de policía. Ante los hechos, el comité organizador dimitió en masa y el nuevo comité realizó una consulta con las Reinas de Guatemala para repensar el concurso. El resultado fue una mayor mayanización tanto en el nombre del mismo, que pasó a escribirse según las normas de la Academia de Lenguas Mayas de Guatemala (Rab 'in Ajaw), como asimismo en la proporción indígena de miembros del jurado y de espectáculos asociados con el concurso.

La Rebelión de las Rabinas fue la materialización de una serie de críticas contra el concurso de Rab'in Ajaw que circulaban desde hacía tiempo en el entorno de las Reinas. Ya en 1978, por ejemplo, la revista Ixim (agosto 1978) había publicado el discurso de la Reina de Quetzaltenango Elba Marina Soch Citalán, en el que criticaba el concurso de Rabin Ajau por utilizar el traje indígena con fines turísticos y folklóricos. En 1994, la representativa de Quetzaltenango, Clara Mariela Limatuj Ixcot, apoyada por la organización que la había postulado, decidió no participar en la elección de Rabin Ajau. En un campo pagado a la prensa comunicó al « pueblo maya » sus razones, basadas en el hecho de que se manipulaba, exhibía y se faltaba el respeto a la mujer maya y se exhibían como típicos y exóticos trajes, bailes y tradiciones para satisfacer intereses económicos y políticos de algunos, incluidos « hermanos mayas que no habían comprendido las auténticas aspiraciones del pueblo ».

El movimiento indígena trajo consigo una resignificación de la pertenencia grupal. Se revisó la historia de las instituciones indígenas, buscando dotarlas de un nuevo sentido cultural acorde con esta movilización. En este contexto, los concursos de Reina Indígena fueron cuestionados y sufrieron una nueva transformación. Uno de los primeros fue el de Quetzaltenango. En 1979 se creó una comisión encabezada por el entonces concejal del Xel-ju, Rigoberto Quemé, para reformar el concurso. Para ello se entrevistaron a personas « con conocimiento del Pop $W u j$ », como el intelectual indígena Adrián Inés Chávez ${ }^{15}$,

15. Maestro originario de San Francisco el Alto (Totonicapán) y fundador de la Academia de la lengua maya-k'iche' en 1959 (Guzmán Böckler 1997, p. XxIII). Es conocido como 
y a « personas mayores de 80 años para establecer la indumentaria de la mujer indígena quetzalteca » (Quemé Sacor 2004, p. 4). El resultado fueron cambios tanto en la forma como en el significado del mismo. Así, se cambió el nombre del evento, que pasó a llamarse Umial Tinimit Re Xelajuj Noj (Hija del pueblo de Xelajuj). También se eliminaron el manto real, la corona y el cetro, que fueron sustituidos por un traje ceremonial consistente en un Nim Pot o huipil largo bordado utilizado por las cofradías quetzaltecas, el Ixcap o cinta de 8 metros de largo enrollada alrededor de la cabeza, el Chachal o medallón y el Pop Wuj. Otros concursos seguirían sus pasos como la Reina Indígena de San Cristobal Verapaz, que en 1986 pasó a llamarse Rixq 'un Kaj Koj (Hija de Kaj Koj) o el de Princesa Maya Cobanera, que en 1994 pasó a llamarse Rab ’in Kob 'an (Hija de Cobán) (Molina 2012).

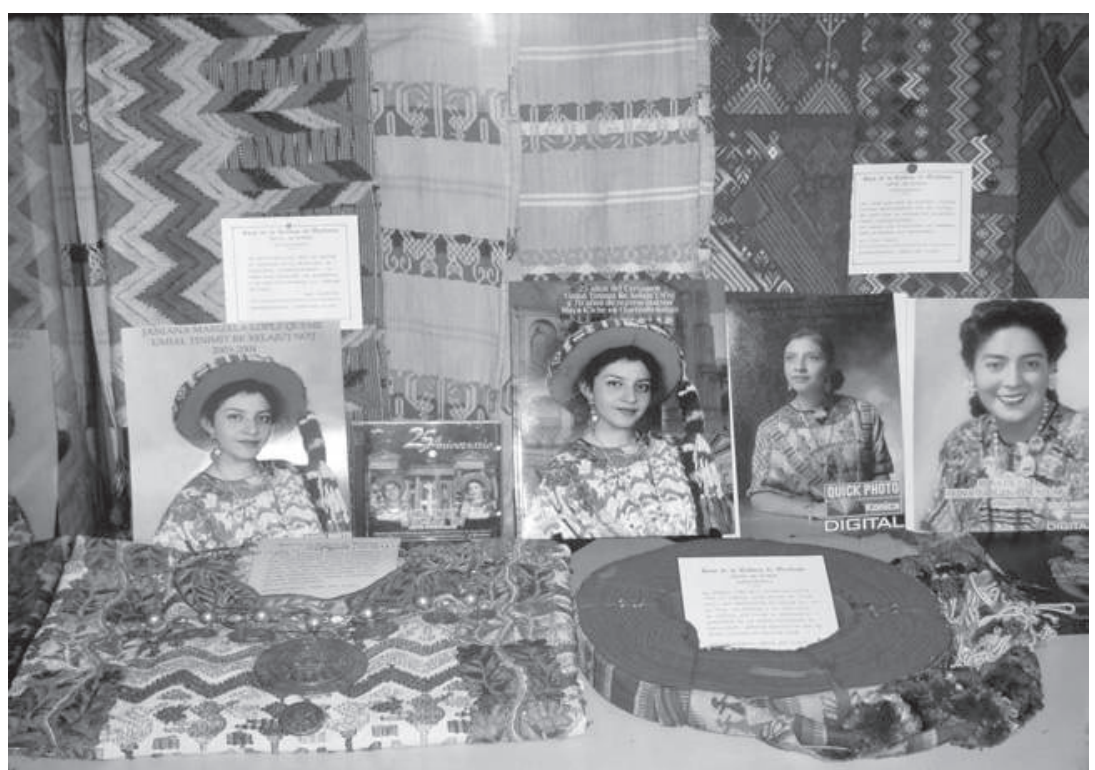

Fig. 4 - Vitrina del Museo de la Casa de la Cultura de Quetzaltenango dedicada a los trajes típicos de Guatemala en la cual podemos observar el Nim Pot, el Ixcap y el Chachal utilizados en el concurso de Umial

Tinimit Re Xelajuj Noj. Fotografía de la autora, Agosto del 2006.

Recordando aquellos tiempos, Rigoberto Quemé (concejal del Xel-ju por aquel entonces y alcalde de Quetzaltenango de 1996 al 2003) afirma que lo que

estudioso de la lengua k'iche', precursor de la normalización lingüística de las lenguas mayas y sobre todo por la publicación de una versión del Popol Vuh que él llamaría Pop $W u j$, escrita a 4 columnas en k'iche' y en castellano. 
más molestaba a los activistas del movimiento indígena era que el concurso se consideraba como una muestra de su cultura, distrayendo de la tarea de « desarrollar la verdadera cultura indígena ». El problema radicaba en saber justamente en qué consistía esta cultura « verdadera », ya que, como veremos, no todo el mundo parecía estar de acuerdo. Las publicaciones de Rigoberto Quemé dejan entrever qué podía ser auténtico para estos activistas:

El evento de Reina Indígena empezó a ser cuestionado desde diferentes perspectivas. ¿Existieron reinas en la cultura Indígena? ¿Somos indígenas o somos mayas? ¿Los elementos simbólicos utilizados en el evento eran propios de la cultura autóctona? ¿Era la municipalidad y sus autoridades quienes debían organizarlo y dirigirlo? ¿Cómo darle más participación al pueblo maya en el evento? (Quemé Chay 2004, p. 2)

La mayanización de las instituciones existentes fue una de las estrategias escogidas para promover el desarrollo de esta cultura « verdadera ». Una mayanización con la que tratarían tanto de resignificar esta pertenencia como también de posicionarse como sus dirigentes legítimos, descalificando a otras posibles representaciones y representantes (Celigueta 2014).

\section{Las Hijas del Pueblo como Representativas Mayas. La representación sentida}

Numerosos autores coinciden en afirmar que, a diferencia de los concursos de belleza oficiales, los concursos indígenas tienen como objetivo celebrar la autenticidad cultural y/o racial (McAllister 1996; Schackt 2005; López García 2015; Konefal 2009). Ante el valor de la apariencia de los primeros, los segundos juzgarían la capacidad para representar una identidad específica según unos cánones que permiten calificar qué muchacha se acerca más y mejor a esta representación ideal. Estos cánones, así como la forma de valorarlos, han ido cambiando con el tiempo por más que mantengan el foco en la autenticidad. Si en 1934 las candidatas de Quetzaltenango debían ser hijas de padre y madre indígenas, en 1999 las bases del concurso de Umial añadían que debían además estar identificadas con la cultura maya-k'iche'. Informaban que el jurado estaría integrado « por personas idóneas con conocimiento sobre la coyuntura que está pasando el pueblo maya » y que, entre otros aspectos, valorarían « la conciencia cultural y la identidad » de la candidata. Ese mismo año, en cambio, las bases del concurso de Señorita Quetzaltenango, en el que compiten las muchachas ladinas de la ciudad, puntualizaban que las candidatas debían medir como mínimo 1,60 metros. ¿En qué consiste entonces esta conciencia cultural y esta identidad? Y, sobre todo, ¿cómo pueden ser representadas en el siglo XXI?

Las ceremonias de elección de $U$ mial Tinimit comienzan con la entrada al Teatro Municipal de cada una de las candidatas bailando el son, mientras el maestro de ceremonias presenta a la joven, sus padres, abuelos, estudios así 
como también al grupo que la ha postulado. A continuación cada una de ellas pronuncia un discurso que suele versar sobre temas de «identidad maya ». En 1999, por ejemplo, se habló de la importancia de la mujer indígena y de los jóvenes en la conservación de esa identidad, pero también de los Acuerdos de Paz y de los derechos indígenas. Otra parte importante de la elección es la presentación de la « estampa cultural » cuyo tema es, según las bases del concurso, « libre siempre y cuando incluya aspectos de la cosmovisión de nuestra cultura maya ». En 1999, las candidatas escenificaron ceremonias mayas y bailaron en medio de mazorcas de maíz y tejidos mayas, mientras se oían voces de fondo recitando trozos del Popol Vuh o explicando lo que significaban los días del calendario maya.

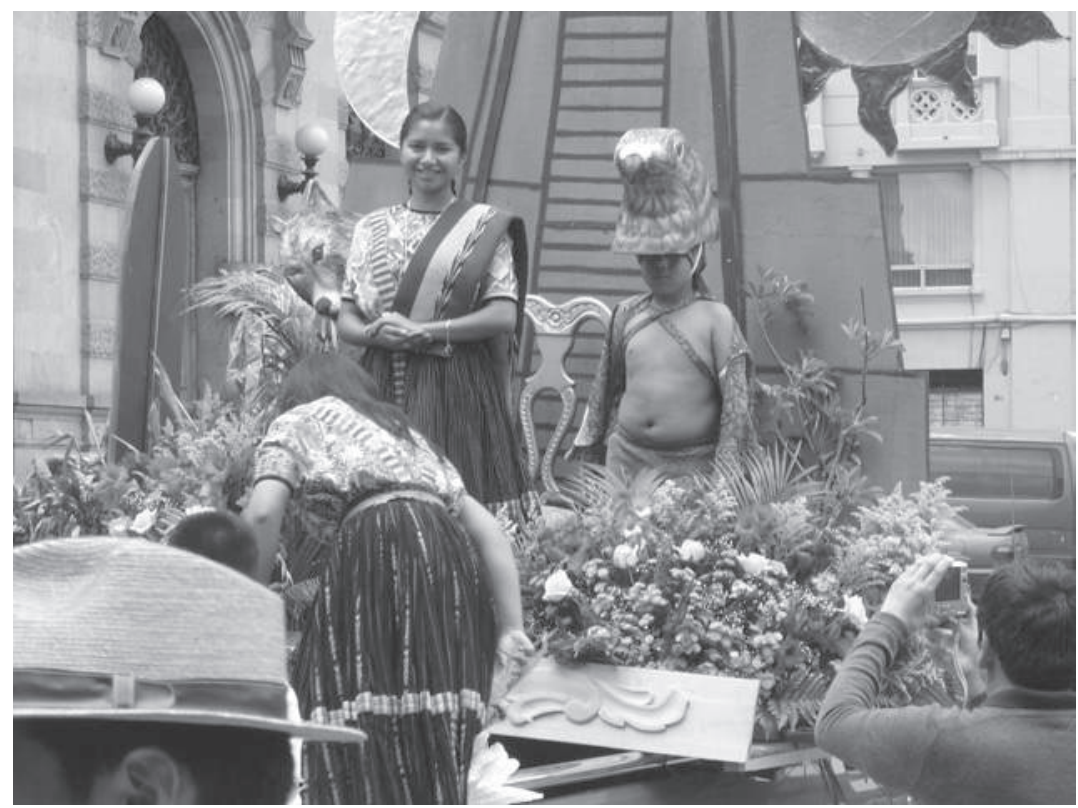

Fig. 5 - Presentación de candidata a Umial en el parque central de Quetzaltenango. Fotografía de la autora, Agosto del 2016.

El desenvolvimiento en el escenario de la candidata, y su forma de ser, hablar y bailar según la elaborada etiqueta k'iche', también son criterios importantes a la hora de elegir a la Umial. Saludar a las autoridades y al público en el orden requerido, llevar correctamente el traje, « bailar bien el son » según el criterio quetzalteco - es decir en una posición semi-inclinada con las manos cruzadas en el vientre y siguiendo un vaivén armónico - son cualidades que no sólo aprecia el jurado sino el público en general. Si como afirma Blacking (1983, p. 95) la danza es un sistema de signos que expresa sentimientos culturalmente 
codificados, el « respeto, el orden, el equilibrio y la armonía », los cuatro valores fundamentales de los mayas según el discurso de una de las candidatas, son encarnados en esta forma de moverse y bailar.

Y sin embargo, estas cualidades no son todavía suficientes para el jurado calificador. En el cambio de siglo, tanto las ganadoras que entrevisté como el concejal de la municipalidad encargado de la organización del concurso parecían tener claro que las candidatas no solo debían representar « bien » su cultura, sino que además debían sentirla. Según la Umial Tinimit de 19992000 , se valora « lo que la persona tenga en mente, que verdaderamente crea en su cultura, no que solamente se aprenda un papel para repetir lo que muchas veces se dice pero sin sentirlo ». El temor es « que sea una representativa que aparente algo que no sea ». El jurado del concurso de Umial en 1999 valoró esta identificación « verdadera » preguntando a la gente de Quetzaltenango, días antes del concurso, si las candidatas usaban cotidianamente el traje indígena.

Las Reinas del siglo xxI, por un lado, deben encarnar la comunidad que dicen representar pero, por otro lado deben tener cierta capacidad para la acción cultural y política (López García 2015, p. 189). Y esta capacidad se valora tanto con los estudios como con la « conciencia » de las candidatas. Si en la década de 1970 los propios activistas del movimiento afirmaban haber utilizado a las Reinas como la única voz autorizada con la cual plantear sus demandas (Konefal 2009, p. 54), a partir de la década de 1990 las mujeres indígenas reivindican su protagonismo en el activismo maya.

En este contexto, las Hijas del Pueblo (que nunca se refieren a ellas mismas con este nombre) rechazan el nombre genérico de Reinas Indígenas y en sus comunicados y discursos y en la prensa reclaman que se las identifique como Representativas Indígenas (o Representativas Mayas cuando no incluyen a mujeres garífunas o xinkas de Guatemala). En una búsqueda por distinguirse de los concursos de belleza usuales, enfatizan el papel representativo (local, regional o nacional) por encima de la valoración de la belleza que según ellas, continua sugiriendo el término de Reina. Molina (2012, p. 91) también argumenta que prefieren el nombre de Representativas porque primero, el concepto de Reina no existe en los idiomas mayas y, segundo, porque en tanto que señoritas electas representan la voluntad popular. Afirman por tanto que su labor actual es de « incidencia política y liderazgo » y se implican no sólo en actos culturales o de beneficencia, sino también en la memoria colectiva del conflicto armado guatemalteco, la defensa del medio ambiente, los derechos de las mujeres indígenas, el rechazo a las transnacionacionales o el juicio por genocidio al General Ríos Montt (López García 2015, p. 189). Así, resuelven, de momento y sobre todo desde esta nueva perspectiva maya, el difícil mandato con el que surgieron los concursos de India Bonita. Por un lado, representan el pasado indígena que los identifica ya sea como nación, como comunidad y/o 
como pueblo y, por el otro, representan las luchas, aspiraciones y demandas de los indígenas del presente.

\section{Las diversas nociones de autenticidad y sus conflictos}

A lo largo de sus casi cien años de existencia, estos concursos se han centrado en mostrar una representación auténtica de lo indio, lo indígena o lo maya. El problema radica entonces en saber en qué consiste esa autenticidad, ya que su definición cambia según los actores y el contexto. Como afirma McAllister (1996, p. 107), la cuestión reside en saber qué es lo auténtico, cómo se autentifica, quién lo hace, en qué contextos y con qué legitimidad. De hecho, como ya hemos señalado, los mayores debates sobre este tipo de concursos tienen que ver con la noción de autenticidad que vehiculan. ¿Muestran los concursos una imagen verdadera, correcta o auténtica de la cultura indígena o maya? ¿Y qué es en cada caso lo verdadero o auténtico? (Schackt 2005, p. 283). Como vimos, cierta noción de autenticidad parece delinearse claramente para la mayoría de los actores indígenas, enfrentándolos en diferentes momentos a las representaciones folklorizantes propuestas por los no indígenas: consiste en reivindicar que son ellos los que deben decidir y encarnar la representación de su propio grupo. Sin embargo, parece más difícil identificar las diferentes nociones de autenticidad que también existen dentro de los grupos indígenas. Este apartado quiere esbozar algunas de ellas a partir de los conflictos que ocurren en estos concursos.

Uno de los momentos más críticos del concurso de Reina Indígena de Xelajú tuvo que ver con el cambio en la forma de elección de la Reina. De elecciones por votación popular en las urnas se pasó en 1964 a elecciones por jurado calificador. Tzunum (1985) y López Quemé (2004) explican la forma en que los simpatizantes se habían acusado mutuamente de manipular los votos durante los años anteriores. Por ello la municipalidad comenzó, para disgusto de muchos, a regular el voto « como en unas elecciones nacionales » (Hupp 1969, p. 138), reclamando la cédula de vecindad de Quetzaltenango o tiñendo el dedo de los votantes para evitar el fraude electoral. En 1964, la comisión organizadora del concurso decidió instaurar el jurado calificador. Según Víctor Manuel Coyoy, octavo concejal de la municipalidad de Quetzaltenango y presidente de la comisión, esto se hizo para evitar « los conflictos entre los simpatizantes y familiares de las candidatas derivados de la confrontación electoral ». No obstante, su interés por eliminar las elecciones se debía también a otros motivos. Las bases del concurso de ese año especificaban que las candidatas debían hablar k'iche' y vestir el traje indígena, justo en un momento en que gran parte de esta generación urbana estaba dejando de hacerlo. Coyoy recuerda: 
En la velada de calificación participó una señorita, de baja estatura, de rasgos mayas clásicos, hablando perfectamente el k'iche', entonces resulta que ella es la triunfadora, pero la generalidad de la población indígena, incluso, ya su mente, no estaba fija en el sentido de belleza que tenían los mayas sino en el sentido de belleza occidental... y mire que la población de Quetzaltenango, sobre todo la indígena, que si más, me pegan.

El conflicto debió de ser importante porque al año siguiente se volvieron a instaurar las elecciones por votación popular. A pesar de todo, el concurso entró en crisis. Durante los años siguientes casi no se presentaron candidatas y en 1970 se estableció definitivamente el jurado calificador que a partir de entonces habría de elegir a las Reinas « auténticas » de Quetzaltenango. Sólo dos años después, en 1972, varios k'iche' que también habían formado parte del entorno de las Reinas crearían una organización electoral indígena, el Xel-ju, que se presentaría a las elecciones municipales de 1974. Según afirma Víctor Manuel Coyoy: «Llegó un momento en que nos dijimos bueno, ya dejémonos de coronar y descoronar Reinas, entremos a algo más fuerte, y fue cuando nace Xel-ju » (Celigueta 2012, 2014).

Este conflicto me sirve para apuntar dos significados posibles de la noción de autenticidad. Por un lado, las elecciones « de verdad », es decir las elecciones municipales o nacionales, se parecían muchísimo a las elecciones de Reina Indígena, en las cuales también se pagaban anuncios de las candidatas en la radio y en los periódicos, se imprimían carteles y volantes con sus fotografías pidiendo el voto o se organizaban actos sociales de presentación de las mismas (Hupp 1969, p. 135-136). Las acusaciones por compra de votos también eran - y son todavía - corrientes en las elecciones municipales o nacionales. No obstante, a estos líderes indígenas les molestaba el conflicto derivado de la confrontación electoral, ya que impedía la unión de la población indígena y su movilización para causas más « verdaderas » como el acceso a la alcaldía municipal. Por otro lado, los líderes indígenas acusan a sus congéneres de no ser capaces de percibir la « auténtica belleza maya ». Es justo en el momento en que se quiere movilizar y resignificar la pertenencia indígena que se eliminan las elecciones de « mentiras » de la Reina, para concentrarse en la elección de « verdad » de sus posibles representantes políticos. No hace falta decir que las Reinas de Quetzaltenango participarían a menudo en las campañas electorales del Xel-ju pidiendo el voto a la población indígena.

Un segundo conflicto que también puede ilustrar esta posible tensión entre las diversas nociones de lo auténtico lo encontramos en el mismo concurso de Quetzaltenango. En 1984 (justo en las bodas de oro del certamen), descontentas con los cambios del concurso, algunas antiguas Reinas crearon una comisión para que el certamen retornara a su nombre original de Reina Indígena. Sostuvieron dos argumentos: por un lado esgrimieron la antigüedad del evento, que le 
confería ya cierta tradición a respetar, y por otro argumentaron que el título de Reina tenía más categoría que el de Umial, a su parecer demasiado « folklórico ». Demasiado folklórico, agregaban, para una época en la que nadie lleva el Ixcap, las mujeres indígenas de Quetzaltenango se tiñen el pelo y llevan zapatos de tacón alto. Reinas y Umiales muestran por lo tanto dos visiones distintas de lo que es auténticamente indígena. Por un lado, se observa cómo la autenticidad - « la verdadera cultura indígena »-, buscada por algunos intelectuales mayas en una historia descolonizada, no sería para las antiguas Reinas « verdadera », por ser demasiado idealizada. Por otro lado, se observa cómo la tradición, ligada con el colonialismo, tendría para estas Reinas un valor en sí misma que no compartiría esta nueva identificación maya (Celigueta 2014). Una identificación, según Bastos y Camus (2003, p. 18), que no es impuesta frente al origen claramente colonial de « indígena », que lleva implícito cierta carga de subordinación. Asumirse como « maya » significa superar la identificación comunal y la de los grupos lingüísticos. Significa orientarse hacia una identidad nacional o de pueblo que identifica una historia y una cultura comunes.

Hacia una dirección similar apuntarían las críticas sobre la autenticidad de la indumentaria y los objetos que portan las diferentes representativas indígenas. En este sentido, si el uso de mantos reales y coronas fue criticado por los mayanistas por no ser un objeto « propio » de su cultura (López García 2015), su sustitución actual por prendas ceremoniales utilizadas particularmente en las cofradías también ha sido censurada por algunos cofrades que consideran una falta de respeto que se utilicen prendas rituales en este tipo de eventos. Como le comenta un informante a Molina (2012, p. 97): « ¿En dónde se ha visto que una cofrade ande corriendo por el parque con una tinaja? »

Parte del malestar de las antiguas Reinas Indígenas de Xelajuj con los cambios ocurridos en el concurso también tiene que ver con el control que ejercen sobre el mismo las organizaciones mayas. Si anteriormente la mayoría de las Reinas formaba parte de cierta clase indígena acomodada de la ciudad, con los cambios ocurridos en el concurso muchas de estas familias creen que ahora tienen pocas oportunidades de ganar. La hija de una de estas familias me comentaba en 1998: «Como se pusieron así los mayas más radicales y nosotras nos maquillamos y sólo usamos el traje en las fiestas, pues ya sabemos que eso no les gusta y no hubiéramos salido. Ellos quieren gente así, natural, natural ${ }^{16}$. » Es decir que ellas no responden a la representación maya propuesta por las organizaciones. Como apunta Rogers (1999, p. 58) en su análisis de los concursos indígenas de Ecuador, los concursos tratan de formalizar cómo deben ser las mujeres indígenas y por extensión la identidad indígena en general, pero esto no tiene por qué ser aceptado necesariamente por la totalidad de la audiencia. Así parecen

16. En Guatemala, a los indígenas se les llama también «naturales », y por ello la expresión « natural, natural » significa aquí indígena auténtico. 
demostrarlo los frecuentes rumores que se escuchan sobre las candidatas y sus familias durante el mes de agosto en Quetzaltenango, durante los días previos y posteriores a la velada de elección: que si se ha comprado al jurado calificador, que si a la gente no le gustó que el jurado no fuera de Quetzaltenango, que si tal candidata tenía el pelo corto y las trenzas eran en realidad postizas, que si la que quedó hablaba mejor pero había otra que en realidad era más bonita, que si ésta no pronuncia bien el k'iche', no es de Quetzaltenango o no tiene apellidos indígenas. Los rumores afectan a todas las aspirantes y cada quién propaga el que más le interesa según la candidata de su preferencia. Como me dijo una señora de una familia de la élite: « los miembros del jurado pueden conocer sobre identidad y cosmovisión pero no sobre qué señorita debe representar a los indígenas de Quetzaltenango ».

\section{Conclusión}

A lo largo de estas páginas hemos visto cómo han ido cambiando representaciones y aspiraciones de los indígenas de Guatemala, pero también hemos demostrado la forma en la cual estos concursos sirven para legitimar su autenticidad. Una autenticidad, eso sí, que siempre es cuestionada en la arena política que constituyen estos - en apariencia - inofensivos concursos de belleza. Los cuerpos vestidos de las Reinas Indígenas con símbolos identificadores - discursos, bailes, mazorcas de maíz o el mismo Popol Vuh - parecen haber sido buenos comunicadores de estas ideas abstractas que son las naciones, las culturas, las razas y los pueblos. Por lo tanto, analizar estos concursos significa la posibilidad de analizar las representaciones de lo indígena en diferentes momentos de su historia. Pero, lejos de imaginarnos a los indígenas como sujetos pasivos que se prestarían a una interesada incorporación folklórica a los Estados-Nación, sabemos que han cuestionado esta folklorización proponiendo representaciones alternativas. Además, estos grupos no son homogéneos y las diferentes formas de representar lo indio, lo indígena o lo maya que hemos identificado en los concursos son buena muestra de ello.

En la actualidad, las Representativas Mayas de Guatemala ya no son Reinas Indígenas ni tampoco son Indias Bonitas. Es decir, ya no son valoradas exclusivamente por su familia o por su belleza. Ya no se valora únicamente que sea bonita sino, como se dice en Guatemala, « que hable bonito ». Ya no se valora una representación elitista de la etnicidad, según la cual las candidatas son hijas de familias respetables y conocidas, sino que se valora la identificación y la conciencia de las concursantes. Por ello es la candidata la que debe de estar identificada con los valores que se quieren resaltar: debe estudiar, debe vestir el traje indígena, debe participar en la vida social de la ciudad y sobre todo debe afirmar públicamente su pertenencia. Así, aunque se trate de resaltar valores comunitarios, étnicos o nacionales, se premian también opciones 
individuales que se muestran como ejemplos a seguir. Ya no basta con ser indígena. Ni tampoco con parecerlo. La representación debe ser verdadera y auténtica según los parámetros de las organizaciones mayas. Debe ser capaz de encarnar cierta noción de pueblo maya, pero sobre todo debe ser capaz de mostrar las aspiraciones de dicho pueblo. ${ }^{17} *$

* Manuscrit reçu en mai 2016, accepté pour publication en mars 2017.

\section{Referencias citadas}

Bastos Santiago y Manuela CAmus

2003 Entre el mecapal y el cielo. Desarrollo del movimiento maya en Guatemala, FLACSO/Cholsamaj, Guatemala.

BLACKING John

1983 «Movement and meaning. Dance is social anthropological perspective. Dance Research », The Journal of the Society for Dance Research, 1 (1), p. 89-99.

Canessa Andrew

2008 «Sex and the citizen. Barbies and beauty queens in the age of Evo Morales », Journal of Latin American Cultural Studies, 17 (1), p. 41-64.

2012 «Gender, indigeneity, and the performance of authenticity in Latin American tourism », Latin American Perspectives, 39 (6), p. 109-115.

Celigueta Gemma

2014 «Representantes y representaciones indígenas en el altiplano occidental de Guatemala », Quaderns-e de l'Institut Català d'antropologia, 19 (1), p. 62-80.

2012 « De Reinas y presidentas: mujeres indígenas y ciudadanía en Guatemala », in Gemma Orobitg y Gemma Celigueta (eds), Autoctonía, poder local y espacio global frente a la noción de ciudadanía. La experiencia indígena y afroamericana desde la interacción politica, social y étnica, Departament d'Antropologia Cultural i Història d'Amèrica i Àfrica, Universitat de Barcelona, Barcelona, p. 307-321.

Cohen Colleen Ballerino, Richard R. WiLk y Beverly Stoeltje

1995 Beauty Queens on the global stage. Gender, contests, and power, Routledge, New York.

GARCíA DE LEÓN Jorge Luis

1978 «El despojo de nuestra identidad en el extranjero por falsos grupos indígenas folkloristas », Ixim, 11, p. 6.

17. Este artículo fue presentado en unas Jornadas de estudio del Laboratoire d'ethnologie et de sociologie comparative de l'université Paris Nanterre, el 11 de diciembre del 2015, como parte del proyecto ANR Fabriq'Am « La fabrique des "patrimoines". Mémoires, savoirs et politiques en Amérique indienne aujourd'hui » (ANR-12-CULT-005). Este artículo forma parte del proyecto «Etnicidad Material: expresiones culturales tangibles y propiedad intelectual » (CSO2015-62723-ERC) financiado por el Ministerio de Economía y Competitividad de España. 
GRANDIN Greg

2007 La Sangre de Guatemala. Raza y nación en Quetzaltenango (1750-1954), Editorial Universitaria, Guatemala.

GuZMÁn BöCKLER, Carlos

1997 « 20 años después: recuerdos de la primera edición del "Pop Wuj” », in Pop Wuj: poema mito-histórico ki-chè. A. I. Chávez, XV-XXIV, Liga maya internacional, San José-Costa Rica.

HANDLER Richard

1986 « Authenticity », Anthropology Today, 2 (1), p. 2-4.

Hupp Bruce F.

1969 The urban indians of Quetzaltenango, Guatemala, Thesis of Master of Arts, university of Texas, Austin.

LÓPEZ GARCÍA Julián

2015 «Reinas indígenas de Guatemala en el siglo xxI: melancolía, orgullo y coraje », in Manuel Gutiérrez Estévez y A. Surrallés (eds), Retórica de los sentimientos. Etnografias Amerindias, Iberoamericana-Vervuert, Madrid, p. 177-231.

López García J., G. Celigueta y L. Mariano

2015 «Las representaciones del indígena desde la antropología en Guatemala », Quaderns de l'Institut Català d'Antropologia, 31, p. 161-182.

LóPez Quemé Janiana Marizela (ed.)

200425 años del Certamen Umial Tinimit Re Xelaju'j N'oj y 70 años de representación maya-k'iche en Quetzaltenango, Kawoq, Quetzaltenango.

McAllister Carlota

1996 « Authenticity and Guatemala's Maya Queen », in Colleen Ballerino Cohen, Richard R. Wilk y Beverly Stoeltje (eds), Beauty Queens on the global stage. Gender, contests and power, Routledge, New York, p. 105-124.

Molina Deyvid

2012 «Apuntes históricos sobre los certámenes de elección y coronación de representativas indígenas en Guatemala », Tradiciones de Guatemala, 78, p. 91-130.

KITE Jillian

2014 The eye of the beholder. Ladino and indigenous pageantry in neocolonial Guatemala, Thesis Master of Arts, Florida Atlantic University.

Konefal Betsy

2009 « Subverting authenticity. Reinas Indígenas and the Guatemalan State, 1978 », Hispanic American Historical Review, 89 (1), p. 41-72.

QuemÉ CHAY Rigoberto

2004 «Importancia histórica, social y política del evento de Reina indígena/ U mial Tinimit Re Xelajuj Noj », in Janiana López Quemé (ed.), 25 años del Certamen de Umial Tinimit Re Xelaju'j N'oj y 70 años de representación maya-k'iche en Quetzaltenango, Kawoq, Quetzaltenango, p. 1-3.

Quemé SACOR Catalina

2004 «Reseña histórica del certamen Umial Tinimit Re Xelaju'j N'oj », in Janiana López Quemé (éd.), 25 años del Certamen de Umial Tinimit Re Xelaju’j $N$ 'oj y 70 años de representación maya-k'iche en Quetzaltenango, Kawoq, Quetzaltenango, p. 4-5. 
QuiJIVIx Ulises

1998 Propuesta de un modelo organizativo, administrativo y gerencial para la Sociedad El Adelanto, Tesis de maestría, Universidad Autónoma de Madrid, Quetzaltenango.

RoGers Mark

1999 «Spectacular bodies. Folklorization and the politics of identity in Ecuadorian beauty pageants », Journal of Latin American Anthropology, 3 (2), p. 54-85.

Ruiz Martínez Apen

2001 «Nación y género en el México revolucionario: La India Bonita y Manuel Gamio ». Signos históricos, 5, p. 55-86.

SCHACKT Jon

2005 «Mayahood Through Beauty: Indian Beauty Pageants in Guatemala », Bulletin of Latin American Research, 24, p. 269-287.

TARACENA Arturo et al.

2002 Etnicidad, estado y nación en Guatemala (1808-1944), Nawal Wuj, Guatemala.

Tzunum Gloria Virginia (ed.)

1985 Historial del certamen de la belleza indígena de Quetzaltenango, Casa Publicitaria Gof, Quetzaltenango.

Velasquez Nimatuj Irma Alicia

2002 La pequeña burguesía indígena comercial de Guatemala, NawalWuj, Guatemala. 
